\title{
REVUE
}

\section{LES PROGRÈS RÉCENTS DANS LA CHIMIE DU LAIT}

\author{
par M. G. GENIN
}

La question du lait et de tous les produits qui en résultent est étudiée depuis fort longtemps par un grand nombre de chimistes qui se sont spázialisés dans les recherches sur ces produits. Pour montrer l'importanse de ces études, il suffit de signaler qu'au cours de l'année 1928, la revue américaine de documentation Chemical Abstracts a publié 336 analyses d'articles ayant trait à toutes les questions se rapportant au lait, à l'analyse du lait, au beurre, aux fromages, à la crèmə glasée, au petit-lait, etc. Dans ce chiffre, on n'a pas tenu compte ni des livres parus sur cette question, ni des brevets.

Un auteur américain, L. S. PALMER (1), a revisé dernièrement les prinzipaux travaux effectués dans ces derniers mois sur l'industrie laitière et nous avons jugé intéressant de reproduire ici les articles analysés par cet auteur. Pour faciliter la lecture de ce travail, les recherches ont été groupées en un certain nombre de titres de paragraphes.

Analyse du lait. - La détermination des matières grasses contenues dans le lait devrait,étant donné l'importance que l'on a attaché à ce problème, être résolue d'une façon définitive depuis déjà longtemps. Néanmoins, cette question est toujours à l'ordré du jour et tout récemment encore Thurston et Peterson (2) ont montré que l'on peut faire de grandes erreurs lorsque l'on recherche dans le petit-lait les matières grasses par les méthodes gravimétriques habituelles par suite de la présence dans ce petit-lait d'un pourcentage élevé de lipides dérivés de la lécithine et du stérol. Depuis fort longtemps déjà, certains chimistes agricoles avaient observé que l'extraction par l'éther d'un certain nombre de produits laitiers ne donne pas le pourcentage des matières grasses contenues dans ces produits, mais ces observations avaient été discutées par les chimistes analystes et la question était restée en suspens.

Les travaux de Thurston et Peterson ont donc apporté plus de prézision sur ce point et ils permettront de mettre en évidence la distinction qu'il faut faire entre les matières grasses contenues dans un produit et l'extrait éthéré, deux quantités qui ne sont pas identiques.

Facteurs influençant la composition du lait. - Les variations qui peuvent se produire expérimentalement ou naturellement dans la composition du lait de vache continuent à faire l'objet de très nombreux travaux dans les différents pays, et à l'heure actuelle 
il semble que ces travaux ont apporté un peu plus de Iumière sur ces questions déjà a nciennes.

Par exemple, Davies et Provan (3) ont montré que les changements qui se présentent dans la eomposition du lait lorsque les animaux sont conduits au pâturage dépendent dans une large mesure de la nourriture donnée aux animaux pəndant l'hiver et plus spécialement de la peoportion də protéines contenues dans ces aliments. C'est ainsi que l'on observe lors du changement de nourriture une augmentation considérable de la proportion de caséine, de phosphore organique et inorganique, de calcium dans le lait lorsque la nourriture d'hiver était pauvre en protéines. Ces changements sont accompagnés d'une augmentation de la quantité de lait produit par l'animal.

Une autrə quзstion très anzienns a été également éslaircie, c'est celle des variations saisonnières de la proportion des matières grasses contenues dans le lait. Weaver et Matthews (4) ont montré, par l'étude de nombreuses statistiques réunies par eux au Collège d'Etat de Iowa, que la proportion de matières grasses contenues dans le lait dépend bэaucoup dəs variations dans la tempárature. Ceci explique le fait bien connu que le lait contient en hiver une proportion de matières grasses bien supérieure à celle qu'il renferme en été.

Par ailleurs, l'expórience a montré que la composition et les propriétés du lait ne sont pas en règle générale modifiées par de légères variations dans la nourriture. Si l'on ostroie à l'animal une nourriture richs en huile, la constitution des matières grasses du lait peut varier, mais la constitution du lait ne se modifie pas. Toutefois, l'addition à la nourriture de quantités importantes d'huile de foie de morue (jusqu'à 250 gr. par jour) pəut diminuer la proportion de matières grasses du 1ait. Cэ fait a été observé par un groupe de chimistes anglais parmi lesquels il faut citer Golding et Drummond. Cette question a de nouveau été étudiée par Matrick (5) qui a montré que l'effet de l'huile de foie de morue est spáaifique et que les autres huiles ne présentent pas ce même effet. A côté de la diminution des matières grasses, MatTick a montré que l'addition d'huile de foie de morue à la nourriture diminue l'acidité titrable du lait, augmente la proportion de calcium et de cendres, diminue la proportion de calcium diffusible et augmente la durée requise pour la coagulation du lait par la présure.

\section{Propriétés chimiques et physiques du lait. - S'SHNECK} (6) a proposé une nouvelle méthode pour étudier le fait queles changements dans le degré de dispersion des constituants du lait sont des facteurs prédominants dans les traitements que peut subir le lait par la suite, tels que écrémage, fabrication du fromage, etc. Cet auteur utilise la diffusion de la lumière pour étudier les modifications de dispersion des matières grasses et de la caséine. 
Parmi les autres propriétés physico-chimiques du lait qui ont été étudiées, il faut citer les propriétés tampon du lait. Comer (7) a utilisé à cet effet le lait de chèvre et a ajouté ce lait à des mélanges de phosphate monosodique, de phosphate disodique et de phosphate trisodique, afin d'étudier le $p$ H des phosphates seuls en fonction du $p$ H des phosphates contenant le lait. Différents auteurs qui ont étudié cette question ont trouvé que e'est la caséine qui exerce une action tampon maximum pour un $p \mathrm{H}$ de 5,2 et que c'est ce constituant du lait qui, à ce point de vue, joue le rôle ca pital. Il est également intéressant de souligner que la paracaséine ne présente pas de maximum analogue à celui qui est montré par la caséine. De plus, la paracaséine diffère de la caséine en présentant plusieurs constantes de dissociation, tandis que la caséine n'en montre qu'une seule dans la région étudiée.

PYNe (8) a publié un important travail relatif à-la viscosité du lait au cours d'une étude faite sur l'àction du sucrate de calcium ou viscogène sur le lait ou sur la erème. A cet effet, il a utilisé un appareil ingénieux permettant de montrer que la grande viscosité que l'on observe lors de l'addition de viscogène est due principalement à la formation d'un précipité de triphosphate de calcium.

Constituants inorganiques du lait. - La découverte récente du rôle joué dans certaines conditions par le cuivre dans le pouvoir nutritif du lait a remis à l'ordre du jourla question du pourcentage du cuivre dans le lait. QUAM et HeLlwig (9) ont montré que le lait normal de vache peut contenir entre 0,37 et $0,50 \mathrm{mmgr}$. de cuivre par litre. Le lait pasteurisé commercial semble plus riche en cuivre que le lait ordinaire et enfin le petit-lait contient de 2,4 à $2,5 \mathrm{mmgr}$.par litre. Des chiffres identiques à ce dernier ont été obtenus pour le lait condensé par suite probablement de la nature métallique des récipients employés dans la préparation de ce lait.

L'acide citrique a pendant très longtemps été considéré comme un constituant très important du lait. Toutefois, JerLor (10) a montré que ce constituant ne se présente dans le lait que le deuxième jour a près la mise à bas. Ce fait peut être intéressant dans l'étude de la synthèse du lait.

Un des problèmes les moins connus semble être celui de l'action de la chaleur sur les composés de calcium et de phosphore contenus dans le lait. Matmick et HalletT (11) a yant maintenu des échantillons de lait pendant 30 minutes à des températures comprises entre $41^{\circ}$ et $98^{\circ} \mathrm{C}$. ont comparé la proportion de phosphore et de calcium totale et diffusible avec celle du lait non chauffé. Ils n'ont pas trouvé d'action sur le phos phore lorsque la température est inférieure à $81^{\circ}$. Au-dessus de cette température, environ $3,5 \%$ du phosphore ditfusible $\epsilon$ st transformé $\epsilon$ n phosphore non diffusible ét cette proportion est pratiquement indé- 
pendante de la tempórature. En ce qui concerne le calcium, l'action de la chaleur semble être plus importante. Entre 57 et $67^{\circ} \mathrm{C}$., environ $0,6 \%$ du calcium diffusible est transformé en calcium non diffusible, cette proportion passe à 2 pour les températures comprises entre 63 et $66^{\circ} \mathrm{C}$. et passe à 2,5 à $3,6 \%$ pour les températures supérieures. Ce chiffre de $3,6 \%$ est un maximum qui ne dépend pas de la température commə dans le cas du phosphore, mais qui a pparâtt à une température inférieure.

Si le calsium diffusible représente également le calcium ionisé, il est difficile de concilier ces modifications relativement peu importantes avec l'action de la chaleur sur le lait, action que l'on a voulu expliquer en faisant intervenir le rôle des sels de calcium.

Constitution chimique des matières grasses du beurre. - Ls travail le plus important fait sur cette question est dû à HiLDITCH et JoNES (12), Ces auteurs ont particulièrement étudié la composition du lait de Nouvelle-Zélande et à cet effet ils ont mis au point de nouvelles méthodes pour déterminer la proportion d'acides gras contenus dans le beurre et également une méthode semi-quantitative permettant de déterminer dans quelles conditions sont combinés les acides gras pour former des glycérides.

Ces travaux ont permis de montrer que la composition approximative des acides gras du beurre est la suivante : acide butyrique, 3 ; ca proique, 2 ; ca pyrilique, 1 ; ca prique, 2 ; laurique, 4 ; myristique, 11 ; palmitique, 28 ; stéarique, 9 ; oléique, 33,5 ; linoléique, 4,5 .

Ces mêmes auteurs ont également trouvé que les matières grasses du beurre contiennent environ $30 \%$ de glycérides saturés, ces glycérides renfermant les mêmes acides gras que ceux que l'on trouve dans la totalité des matières grasses du lait et environ dans les mêmes proportions. Ces matières grasses renferment également $36 \%$ de mélanges de glycérides disaturés, le restant étant constitué par des glycérides monosaturés.

Composition chimique du beurre.-Quoi qu'il soit possible actuellement de fabriquer le beurre dans des conditions telles que l'on puisse obtenir des produits d'une uniformité remarquablę, un certain nombre de problèmes très intéressants se posent ayant en vue l'étude de l'influenze de la concentration des différents constituants de la crème sur la fabrication du beurre. VAN DAM et HoLwERDA (13) ont étudié en Hollande plus spécialement l'intluence de la variation de la concentration de la caséine, de l'albumine et de l'acidité. Il est étonnant d'apprendre, comme suite à ces travaux, que les crèmes renfermant un pourcentage élevé de caséine se barattent plus facilement a vec moins de matières grasses que les crèmes ayant un pourcentage bas de caséine. L'inverse est vrai pour les crèmes contenant un faible pourcentage d'albumine. 
En ce qui concerne l'influence de l'acidité, l'expérience a montré que la diminution du $p \mathrm{H}$ de 4,68 à 4,38 diminue la durée du barattage et également la perte en matières grasses, la crème à basse teneur en acide donnant un petit-lait renfermant $0,54 \%$ de matières grasses, alors qu'une crème à haute teneur en acide donne un petit-lait renfermant $0,37 \%$ de matières grasses.

Chimie de la caséine. - Les chimistes biologistes seront intéressés par les travaux de LINDERSTROM-LANG (14) sur l'individualité de la caséine qui a pendant très longtemps été considérée comme une substance spécifique. Dans ses travaux les plus récents, cet auteur a montré que la caséine n'est pas constituée par une substance individuelle. Cette évidence $\epsilon$ st basée sur le fait que la caséine pure peut être soumise à un fractionnement au moyen d'alcool à $60 \%$ acidifié contenant 0,001 à $0,002 \mathrm{~N} \mathrm{HCl}$. Les différentes fractions ainsi obtenues présentent un pourcentage de phosphore variant de 0,15 à $1 \%$, un pourcentage de tryptophane variant de 1,4 à $2,3 \%$ et un pourcentage de tyrosine variant de 3,8 à $6,1 \%$, le pourcentage d'arginine et de lysine ne variant que dans de très faibles proportions.

Si l'on mélange les diverses fractions obtenues avec la portion non dissoute, on obtient une caséine dont les propriétés chimiques et physiques sont identiques à celles de la caséine initiale. Ces résultats, s'ils sont vérifiés, permettront dans beaucoup de eas d'expliquerles différences observées par certains auteurs dans les propriétés physicochimiques de la caséine. Il reste à déterminer si cette pluralité de la caséine est caractéristique de la caséine telle qu'elle existe dans le lait, ou si elle est simplement une caractéristique de la substance après isolement.

Chimie de l'action de la présure. - La substance qui résulte de l'action de la présure sur la caséine $\epsilon$ st connue sous le nom de paracaséine, cette réaction n'€st pas réversible. Les plus importants travaux publiés sur la question de la présure ont pour base la nature de cette transformation; est-elle purement biochimique, ou au contraire prinsipalement colloïdale, les deux types de réaction coexistent-ils? quel est le plus important? Des travaux récents ont apporté quelques lumières sur cette question.

Du point de vue chimique, Porcher (15) a montré que l'hypothèse ancienne de Hammarstein, tout au moins en partic, est correcte et que la présure entraîne une hydrolyse partielle de la molérule de caséine lors de la modification de la caséine en paracaséine. Porcher a trouvé également qu'il existe une petite fraction (environ $4 \%$ ) de l'azote qui reste dans tous les cas non coagulée et qui peut être dans certains cas résupérée sous la forme d'une substance définie ayant les propriétés de la protéose. Il reste néanmoins à prouver si ce scindement de la molé- 
cule est essentiel pour la formation de la paracaséine ou cette hydrolyse est accidentelle et montre l'analogie qui existe entre la présure et la pepsine.

Les prinzipales différences existant entre la caséine et la paracaséine peuvent s'observer surtout dans la solubilité et la capacité de combinaison aux acides et aux bases. Ces différences, qui ont déjà été montrées par PALMer et RichaRdson (16), ont été récemment confirmées par Pertzoff (17) et il semble douteux que la différence de $4 \%$ dans le pourcentage d'azote soit suffisant pour expliquer les différenzes connues entre les propriétés de la caséine et de la paracaséine.

Un des problèmes qui est en rapport a vee celui de la coagulation du lait par la présure est celui qui a pour objet l'action de la chaleur sur la rapidité de coagulation du lait et sur le caractère du coagulum. Un certain nombre de faits nouveaux ont été montrés par MatTick et Halletr (18). Ces auteurs ont chauffé différents échantillons de lait pendant 30 minutes et à des températures comprises entre 41 et $98^{\circ} \mathrm{C}$. et ils ont observé le temps nézessaire pour la coagulation par la présure dans des conditions standards et après refroidissement à $29^{\circ}$. Ils ont observé que si le chauffage a été effectué entre 41 et $60^{\circ}$, la coagulation est accélérée à condition que l'action de la présure s'exerce immédiatement après le refroidissement, au bout d'un certain temps en effet, le lait reprend sa durée normale de coagulation. Si le lait a été chauffé au-dessus de $63^{\circ}$, il se produit un effet retardateur sur la coagulation. Lorsque le lait a été chauffé à $92^{\circ} \mathrm{C}$., le lait ne se eoagule pas, même s'il reste refroidi pendant 45 minutes.

Il semble évident que ces effets très importants ne sont pas expliqués par l'hypothèse ancienne qui admettait que l'influence de la chaleur sur la coagulation du lait par la présure était due à une élimination des sels de calcium solubles. Les récents travaux de MAтTICK et Hallett montrent que la quantité de calcium diffusible éliminée du lait par la chaleur atteint un maximum pour une température comprise entre 63 et $66^{\circ}$. Il semble que ces phénomènes montrent un exemple très frappant de l'hystérésis dans les systèmes colloïdaux et que l'effet de la chaleur sur le lait est dû à une action de la chaleur sur le constituant colloïdal du lait le plus important : la caséine.

On peut donc se demander jusqu'à quel point l'action de la présure peut être expliquée du point de vue colloïdal. Richardson et Palmer (19) ont donné une explication en se plaçant au point de vue électrocinétique. Ils ont observé que les solutions de caséinate de calcium à un $p$ H égal ou inférieur à 6,8 montrent un effet retardateur sur la vélocité cataphorétique de leurs protéines lorsqu'elles ont été placées au contact de présure active. Au-dessus de ce $p H$, il n'y a aucun effet sur la vélocité eataphorétique. Ces chercheurs ont montré de plus que pour un $p \mathrm{H}$ inférieur à 6,9 , les solutions de présure seule présentent une 
cata phorèse de leurs constituants colloïdaux qui est en direction opposée à celle de la caséine, tandis qu'au-dessus de ce $p H$, les colloïdes de la présure se déplacent dans la même direction que la caséine. Ces faits montrent donc que la présure active porte des charges électriques opposées à celles de la micelle de caséine. Il apparaît donc que l'un des effets de la présure, sinon le principal, est dû au pouvoir de ce produit de réduire la charge électrique de la caséine. Par suite, comme la charge de la caséine est négative, le micelle de caséine sera rendu plus sensible à l'action des cathions chargés positivement et principalement des cathions divalents qui entraîneront la coagulation.

On peut expliquer de même l'action de la chaleur. Nous avons vu que la chaleur augmente la vélocité cataphorétique des solutions de caséine, donc la charge électrique du micelle. Comme la présure réduit cette charge, il s'ensuit que le lait préalablement chauffé présentera une sensibilité différente à l'action de la présure de celle du lait non chauffé.

\section{RÉFÉRENCES}

[1] Ind. Eng. Chem., t. XXII, p. 39, 1929.

[2] Journ. Dairy Sc., t. XI, p. 270, 1928.

[3] Welsh. Journ. Agr., t. IV, p. 114, 1928.

[4] Iowa Agr. Expt. Sta. Bull., CVII, 1928.

[5] Biochem. Journ., t. XXII, p. 144, 1928.

[6] Milchwirtschaft. Forsch., t. VII, p. 1, 1928.

[7] Bull. Soc. Ital. Biol. Sper., t. III, p. 908, 1928.

[8] Journ. Agr. Sc., t. XIX, p. 463, 1929.

[9] Journ. Biol. Chem., t. LXXVIII, p. 681, 1928

[10] Svensk Lakaretidning, mai 1929.

[11] Journ. Agr. Sc., t. XIX, p. 452, 1929.

[12] Analyst, t. LIV, p. 75, 1929.

[13] Le Lait, t. VIII, pp. 698, 768, 897, 1928.

[14] Z. Physiol. Chem., t. CLXXVI, p. 78, 1928.

[15] Chimie et Industrie, t. XIX, pp. 589-809, 1928.

[16] Colloid Symposium Monograph, vol. III, p. 112, 1925.

[17] Journ. Gen. Physiol., t. XI, p. 239, 1928.

[18] Journ. Agr. Sc., t. XIX, p. 452, 1929.

[19] Journ. Phys. Chem., t. XXXIII, p. 557, 1929.

\section{BIBLIOGRAPHIE ANALYTIQUE}

\section{LES LIVRES}

Stronl (A.), professeur de physique médicale à la Faculté de médecine de Paris, et collaborateurs : MM. BLANCHetik̀re, Dognon, Fabre, Lescadr, SANní et Wurmser. - Leçons de physicochimie à l'usage des médecins let des biologistes. - Un vol. broché, 284 pages, Masson et Cie Ed., Paris, '1930, 40 francs. 